\title{
PROFILING BELL'S PALSY BASED ON HOUSE-BRACKMANN SCORE
}

\author{
Insu Song ${ }^{1}$, John Vong ${ }^{1}$, Nguwi Yok Yen ${ }^{1}$, Joahchim Diederich ${ }^{2}$ and Peter Yellowlees ${ }^{3}$ \\ ${ }^{1}$ School of Business (IT), James Cook University, Singapore campus, \\ 600 Upper Thomson Road, Singapore, \\ insu.song@jcu.edu.au,john.vong@jcu.edu.au,nguwi.yokyen@jcu.edu.au \\ ${ }^{2}$ University of Melbourne, Australia \\ j.diederich@unimelb.edu.au \\ ${ }^{3}$ Department of Psychiatry and Behavioral Sciences, UC Davis \\ peter.yellowlees@ucdmc.ucdavis.edu
}

\begin{abstract}
In this study, we propose to diagnose facial nerve palsy using Support Vector Machines (SVMs) and Emergent Self-Organizing Map (ESOM). This research seeks to analyze facial palsy domain using facial features and grade the degree of nerve damage based on the House-Brackmann score. Traditional diagnostic approaches involve a medical doctor recording a thorough history of a patient and determining the onset of paralysis, rate of progression and so on. The most important step is to assess the degree of voluntary movement of the facial nerves and document the grade of facial paralysis using HouseBrackmann score. The significance of the work is the attempt to understand the diagnosis and grading processes using semi-supervised learning with the aim of automating the process. The value of the research is in identifying and documenting the limited literature seen in this area. The use of automated diagnosis and grading greatly reduces the duration of medical examination and increases the consistency, because many palsy images are stored to provide benchmark references for comparative purposes. The proposed automated diagnosis and grading are computationally efficient. This automated process makes it ideal for remote diagnosis and examination of facial palsy. The profiling of a large number of facial images are captured using mobile phones and digital cameras.
\end{abstract}

\section{Introduction}

In recent years, machine learning techniques, such as support vector machines (SVMs), have shown significant potential in enhancing medical practice [1]. Usually expert medical doctors like psychiatrists, consciously and unconsciously analyze observable symptoms, such as the patients' language, for examination purposes using diagnostic classification systems, such as DSM IV [2]. To provide a more objective clinical diagnosis, SVMs have been applied to conversations of patients and clinicians [3]. In this paper, we investigate how SVM can be used to diagnose facial nerve palsy. In particular, Bell's palsy or idiopathic facial paralysis (IFP) is a common disease, affecting all ages, regardless of gender and has a high rate of remission [4]. It has been reported as an adverse event following immunization (AEFI). Admittedly there is confusion over the definition of Bell's palsy. According to Webster's Medical Dictionary [5], Bell's palsy is the paralysis of facial nerve, the nerve that supplies 
the facial muscles on one side of the face. The facial nerve controls the muscles of facial expression. Paralysis results in a lack of facial expression. This is not only an aesthetic issue, but also affects the patient, making it hard to communicate effectively [6]. The cause of facial nerve paralysis is thought to be caused by virus . The disease typically starts suddenly and causes paralysis of muscles on one side of the face, on which the facial nerves are affected [5]. Patients often complain of having a peculiar sensation on the side of the face where paralysis is taking or taken place. . This is usually attributable to faulty signals from the paralyzed muscles [7]. About $80 \%$ of patients recover within weeks to months. The diagnosis of Bell's palsy is important in order to provide timely and effective treatments.

Diagnosis of IFP poses a greater challenge in the case of facial paralysis in children. It is more difficult to perform an electrodiagnostic test of facial function on infants or young children than in adults because children are often uncooperative and has limited ability to assist [8]. This makes an automated diagnosis highly desirable. Kasse et al. [4] reviewed 1,521 clinical cases of Bell's palsy and their occurrence according to sex, age, paralyzed side, installation mode, previous symptoms, associated symptoms, and minimal electrical stimulation tests. They concluded that clinical factors and minimal electrical simulation tests could be used to indicate the prognosis of facial palsy when electroneurography is not available.

The standard method of measuring facial nerve functions is using the House-Brackmann facial nerve grading system, which was introduced in 1983 [9], and endorsed by the Facial Nerve Disorders Committee of the American Academy of Otolaryngology in 1984. The grading scale has been thought to accurately describe a patient's facial function and to monitor their status over time to assess the course of recovery and the effects of treatment. It is also a scale that can be used rapidly enough to be useful for clinical practice [10]. However, it was developed as an overall scale, with the objective of placing patients in general categories. It has wide applications and is found to be reliable. Table 1 shows the House-Brackmann Grading System (HBGS). It has been noted that the HBGS is based on the functioning of eyes closure, mouth and forehead movement to determine estimated func- tion. The House-Brackmann Score further grades the degree of nerve damage in facial nerve palsy from $0 \%$ (Grade VI) to $100 \%$ (Grade I).We propose to grade Bell's palsy based on the HBGS using Support Vector Machines (SVMs) [11] and ESOM [12] which are described in Section II.

This automated assessment method of Bell's palsy can be implemented on smart phones, such as Android mobile devices. The rise in the usage of mobile phones now enables us to collect high quality image data conveniently at home, even by parents of children. In particular, digital cameras of cell phones provide easy to use interfaces for capturing useful information about general well being of individuals. Mobile devices have been used in quick diagnosis of health conditions [13], health social networks [3], and improving livelihood of rural areas in developing countries [14]. This rapid access to mobile devices worldwide now opens up new opportunities to provide more convenient and cost effective means of diagnosing various health conditions associated with Bell's palsy, such as cold and flu.

\section{Background}

The following section provides a brief overview of the main techniques, focusing on telemedicine, automated health assessment technology, support vector machines (SVMs), Emergent SelfOrganizing Map (ESOM), and the significance of generating human comprehensible explanations from classifiers. It also seeks to explain the decision-making process of a machine learning system to a human user who may not be a subject matter expert or familiar with methods in information technology.

\subsection{Telemedicine}

Telemedicine is the use of communication technologies, such as telephone, video conferencing, or the Internet, to provide and support health care to remote regions. Telemedicine was initially developed to provide health care to rural and poor populations [15], but distance is no longer the major factor that defines the term. In fact, the applications have been extended to all types of health care including psychiatry consultations in larger cities 


\begin{tabular}{|c|c|c|}
\hline Face & Grade & Characteristics \\
\hline \multirow[t]{6}{*}{ Forehead } & I. Normal & Normal function \\
\hline & II Mild Dysfunction & Slight weakness to good function \\
\hline & III. Moderate Dysfunction & $\begin{array}{l}\text { Noticeable slight to moderate } \\
\text { movement }\end{array}$ \\
\hline & IV. Moderately Severe Dysfunction & $\begin{array}{l}\text { Obvious weakness or disfiguring } \\
\text { asymmetry }\end{array}$ \\
\hline & V. Severe Dysfunction & Barely perceptible motion \\
\hline & VI. Total Paralysis & No movement \\
\hline \multirow[t]{6}{*}{ Eye } & I. Normal & Normal function \\
\hline & II Mild Dysfunction & Complete closure with minimal effort \\
\hline & III. Moderate Dysfunction & $\begin{array}{l}\text { Obvious weakness, eye closure with } \\
\text { effort }\end{array}$ \\
\hline & IV. Moderately Severe Dysfunction & Incomplete eye closure \\
\hline & V. Severe Dysfunction & Barely perceptible eyelid movement \\
\hline & VI. Total Paralysis & No movement \\
\hline \multirow[t]{6}{*}{ Mouth } & I. Normal & Normal function \\
\hline & II Mild Dysfunction & $\begin{array}{l}\text { Slight asymmetry or weakness of } \\
\text { mouth movement }\end{array}$ \\
\hline & III. Moderate Dysfunction & Obvious but no disfiguring weakness \\
\hline & IV. Moderately Severe Dysfunction & Asymmetry at rest \\
\hline & V. Severe Dysfunction & Barely perceptible mouth movement \\
\hline & VI. Total Paralysis & No movement \\
\hline
\end{tabular}

Table 1. House-Brackmann Grading System

[16-18]. The greatest benefit is to serve the rural poor, , because it provides easy access to medical services. Another current driving force is to make health care more cost-effective, affordable, efficient, and convenient for both health care providers and consumers. For instance, Harrison [19] analyzed teleconferences between doctors for improving communication between primary and secondary health care, Yellowlees [18] reported case studies of telepsychiatry through videoconferencing and their potential to improve patient care and satisfaction, and reduce emergency department overcrowding. Frantzidis, et al. [20] developed a remote monitoring system for the elderly and chronically ill. Tang, et al. [21] used a multimedia system to improve the regular and timely consumption of medication by the elderly. The growing percentage of aged populations indicates that this form of telemedicine will become an important research area for many developed countries.

Almost all technologies we have reviewed here can be used jointly with tele-mentalhealth technology. For instance, it will be possible to use automated speech analysis methods (e.g., [3, 22-24] while the patients are being interviewed by psychiatrists to provide objective analysis of speech and language disorder or to monitor improvements in mental health conditions.

Much needed research is now being devoted to the development of more sophisticated Telemedicine technologies that integrate various technologies, such as mobile breathing sound analyser [25] and recommender system [3]. The papers review some of these technologies, with the aid of quantitative evaluation, rather than subjective qualitative evaluations [18]. They also examined the use of modern communication technologies for telehealth and contemporary issues on health, such as how technology is changing the way health care services are delivered and how the relationship between patients and health care providers is changing.

\subsection{Automated Health Assessment Sys- tems}

Many health assessment methods are time consuming and highly subjective. To improve efficiency as well as to provide more objective assessments, various automated health assessment methods have been developed. The methods can be broadly classified based on the type of data col- 
lected and analyzed. We shall now review image analysis-based methods.

Cowie, et al. [26] reviewed the use of image analysis techniques in detecting emotional cues from facial expressions (still face images) and gestures (movements of facial features). According to [26], there are two emotion detection paradigms, namely detecting (seven) typical expressions and that of detecting non- archetypal expressions (e.g., adapted, falsified, or mixed expressions). The majority of existing literature and available data clearly focus on the method of detecting typical expressions (e.g., [27-29]. Much work needs to be done on the detection of deeper non-archetypal emotional expressions and gestures. These approaches now focus on the detection of action units (AU) defined by [30]. For instance, Valstar and Pantic [31] used a facial point detector based on Gabor-feature-based boosted classifiers, achieving AU recognition rate of $95.3 \%$. With regards to the application of facial expressions detection method on the diagnosis of mental health, Stone and Wei [32] used a facial expression detector to measure cognitive load, which is normally assessed by EEG or EMG measurements. However, there is little research on the use of facial emotional detection methods for clinical diagnosis of mental health problems. The possible reason being image processing techniques are more difficult than other techniques, such as sound processing. Significant variations on emotional expressions also make it difficult to develop reliable measurements that are linked to underlying psychological problems.

Instead of relying on face detection methods, Nambu, et al. [33] developed a simple image processing technique for monitoring behaviours and diagnosing poor health in the elderly. The researchers developed an automatic diagnosis system that can assess physical conditions as well as mental conditions from behaviours of the elderly while they watched television. . They used only the recordings of the start time of watching TV obtained from a running monitor of the television. Initially, they tried to utilize various other sensors, such as a running monitor of electric appliances or door switches, but found that the collected data was difficult to analyze objectively. The data appeared to be uncorrelated. The start times of watching TV are recorded by dividing each day into $15 \mathrm{~min}$ in- tervals. An interval is given a certain value, say 1 , when an elderly participant starts the TV in the interval and another value, say 0 , otherwise. This way, a $30 \times 96$ pixel image could be constructed for observing behaviours of elderly watching TV. Their hypothesis was that the subjects watched the television at roughly fixed times if they were healthy. To measure productiveness of their TV watching behaviour, the researchers measured non-randomness of the patterns on the observed behaviour using the maximum entropy method (MEM). The image compression rate was measured as an indication of the health condition. The lower compression rate (larger size of compressed image) was used as an indication of unhealthy condition.

\subsection{Support Vector Machine}

The Support Vector Machine was originally a linear classifier based on optimal hyperplane algorithm developed by Vapnik in 1963[34]. In 1992, Bernhard Boser, Isabelle Guyon and Vapnik successfully applied kernel method to a maximummargin hyperplane and build a non-linear classifier [35] \{ $\{$ Boser, $1992 \# 169$;Boser, $1992 \# 11$ \} \}. In 1995, Cortes and Vapnik[36] suggested a modified maximum margin classifier called soft margin classifier that allows misclassified data with a soft margin. If there is no hyperplane that can separate the data into two classes, the soft margin classifier selects a hyperplane that separates the data as cleanly as possible with maximum margin.

Burges [37] provided a tutorial on Support Vector Machines and its application on pattern recognition. Joachims [38] in 1997 published that SVM outperformed all other major classifiers in text classification. Although SVM is naturally binaryclassifier, Rennie and Rifkin [39] have applied SVM in multi-class text classification and compared with Nave Bayesian classifier. In their experiment, SVM gave much lower error scores than Naive Bayes.

SVMs are based on the structural risk minimization principle in order to overcome the overfitting problems. Support vector machines find the hypotheses out of the hypothesis space $H$ of a learning system which approximately minimizes the limits on the actual error by controlling the experimental error using training samples and the complexity of the model using the VC-dimension of $H$. SVMs are 
very universal learning systems [38]. In their basic form, SVMs learn maximal margin hyperplanes (linear threshold functions). A hyperplane can be defined by a weight vector w and a bias $b$ :

The corresponding threshold function for an input vector $\mathrm{x}$ is then given by:

$$
f(x)=\operatorname{sign}(w \cdot x+b)
$$

However, it is possible learn polynomial classifiers, radial basis function (RBF) networks and three or more layered neural networks by mapping input data $x$ to some other (possibly infinite dimensional) feature space $\varphi(x)$ and using kernel functions $K\left(\mathrm{x}_{i}, \mathrm{x}_{j}\right)$ to obtain dot products, $\varphi\left(x_{i}\right) \cdot \varphi\left(x_{j}\right)$, of feature data.

\subsection{Emergent Self-Organizing Map}

Emergent Self-Organizing Map (ESOM) [40], also referred to as a Kohonen map having a large number of map neurons, is a technique using nonlinear projection neurons arranged in a map $M$, usually in $2 \mathrm{D}$ (i.e., $M \subset \mathfrak{R}^{2}$ ), for low-dimensional visualization of high dimensional data. Two types of ESOM grid structures are commonly used: Hexgrid (honeycomb like) and Quadgrid (as a lattice) maps.

ESOM usually forms a (2D) grid of prototype neurons, which are represented using highdimensional vectors having the same size as the number of features of input data. The density of data in the vicinity of the neurons, and the distances between the neurons, are taken into account for better visualization. For example, an ESOM map can be visualized using

1. Distance-based Visualization called U-Map (from U-Matrix)[41];

2. Density-based Visualization called P-Map (from P-Matrix)[42];or

3. Distance- and Density-based Visualization called $\mathrm{U}^{*}$-Map (which combines the $\mathrm{U}$ and $\mathrm{P}$ map) [43].

The different types of maps show s how the high dimensional data space on a floor space layout using a landscape like visualization for distance and/or density structure of the high dimensional data space. Structures emerge on top of the map by the collision of many neurons during the learning phase.
These emerging structures are the main concept of ESOM. It can be used to achieve visualization, clustering, and classification. This can be obtained by the following.

\begin{tabular}{llllll}
\hline Grade & $\begin{array}{l}\text { Generaliza } \\
\text { tion }\end{array}$ & Recall & $\begin{array}{l}\text { F- } \\
\text { Measure }\end{array}$ & GMts & GMtr \\
\hline I. & $59.38 \%$ & $91.43 \%$ & $72.00 \%$ & $59.54 \%$ & $93.54 \%$ \\
II & $93.75 \%$ & $97.14 \%$ & $95.42 \%$ & $80.23 \%$ & $98.37 \%$ \\
III. & $87.5 \%$ & $100.00 \%$ & $93.33 \%$ & $62.06 \%$ & $100.00 \%$ \\
IV. & $90.63 \%$ & $100.00 \%$ & $95.08 \%$ & $56.73 \%$ & $100.00 \%$ \\
V. & $68.75 \%$ & $97.14 \%$ & $80.52 \%$ & $69.63 \%$ & $95.74 \%$
\end{tabular}

Table 2. Performance Metrics Using Emergent Self-Organizing Map

Let $m: D \rightarrow M$ be a mapping from a high dimensional data space $D \subset \Re^{n}$ onto a finite set of positions $M=\left\{n_{1}, \ldots, n_{k}\right\} \subset \mathfrak{R}^{2}$ arranged on a grid. Each position has its two dimensional coordinates and a weight vector $\boldsymbol{w}=\left\{w_{1}, \ldots, w_{k}\right\}$, which is the image of a Voronoi region in $D$ : the data set $E=\left\{x_{1}, \ldots, x_{d}\right\}$ with $x_{i} \in D$ is mapped to a position in $M$ such that a data point $x_{i}$ is mapped to its best-match $b_{m}\left(x_{i}\right)=n_{b} \in M$ with $d\left(x, w_{b}\right) \leq d\left(x, w_{j}\right)$; $\forall w_{j} \in \mathbf{W}$, where $d$ is the distance on the data set. The set of immediate neighbors of a position $n_{i}$ on the grid is denoted by $N(i)$.

The clustering of ESOM is based on the $\mathrm{U}^{*} \mathrm{C}$ clustering algorithm described by[44]. Consider a data point $x$ at the surface of a cluster $C$, with a best match of $n_{i}=b m(x)$. The weight vectors of its neighbors $N(i)$ are either within the cluster, in a different cluster or interpolate between clusters. Assume that the inter cluster distances are locally larger than the local within-cluster distances, then the U-heights in $N(i)$ will be large in such directions which point away from the cluster $C$. Thus, a so-called immersive movement will move away from cluster borders. This immersive movement performed starts from a grid position, keeps decreasing the U-matrix value by moving to the neighbor with the smallest value, then keeps increasing the P-matrix value by moving to the neighbor with the largest value. The details of this clustering algorithm can be referred to [44]. 


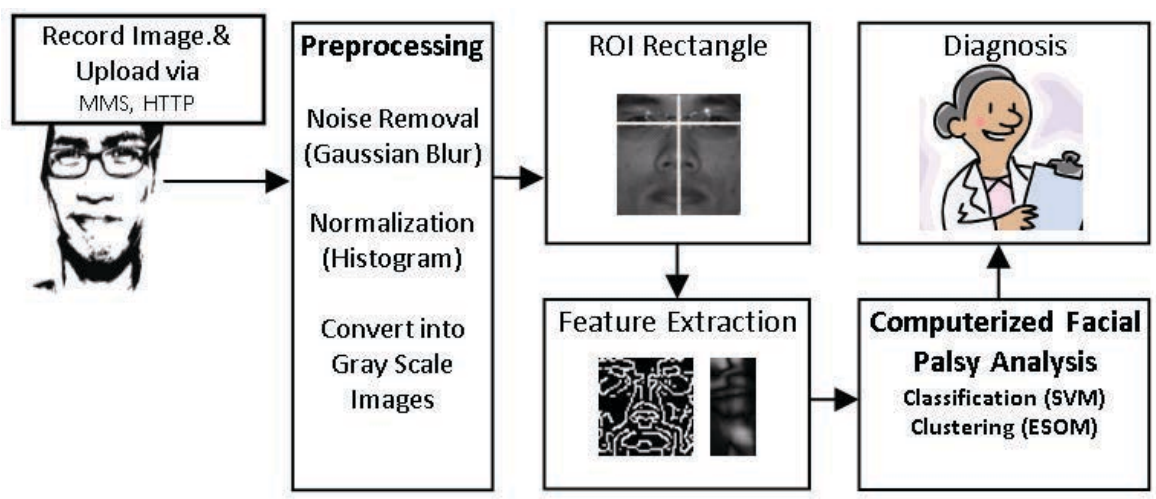

Figure 1. The overall process of automated House-Brackmann Grading of face images.

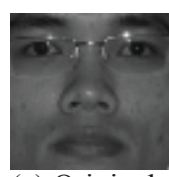

(a) Original

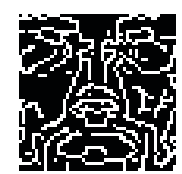

(b) Edge

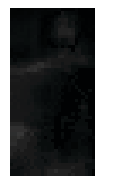

(c) Difference

Figure 2. Intermediate Images of normal face. (a) Original face. (b) Significant face edges (c) Difference between left and right face.

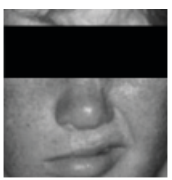

(a) Original

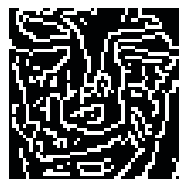

(b) Edge

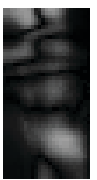

(c) Difference

Figure 3. Intermediate Images of palsy face (Grace V). (a) Original face. (b) Significant face edges (c) Difference between left and right face.

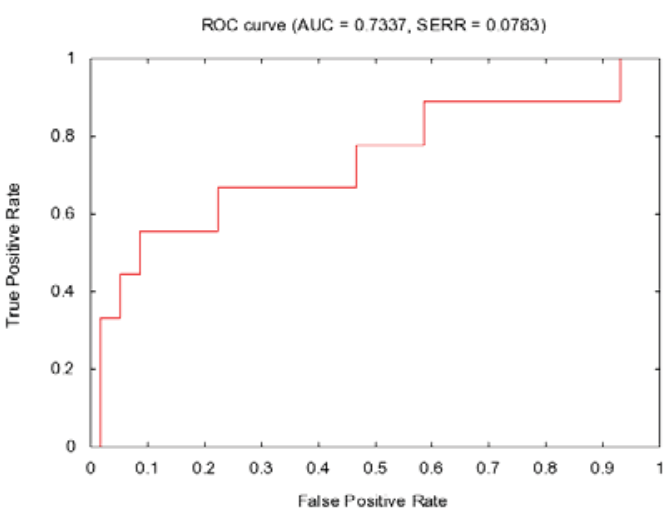

(a)

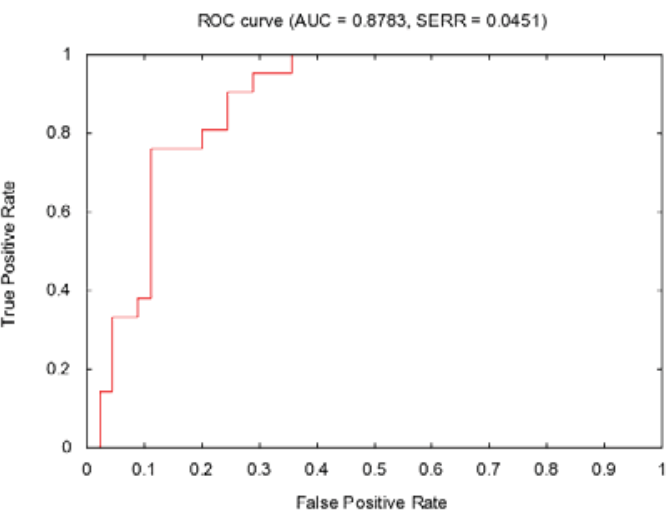

(b)

Figure 4. ROC using (a) Ten principal components (b) Hamming Distances of left face and right face 


\subsection{Explanation - The foundation}

The ability to explain the outcomes is important where high security is required, such as medical applications. Although sophisticated machines learning algorithms, such as support vector machines (SVMs) and Emergent Self-Organizing Map (ESOM) have shown superior performance in a range of classification and regression tasks. SVMs, such as artificial neural networks (ANNs), make it difficult to explain the outcome. There is a significant literature on obtaining human-comprehensible rules from SVMs and ANNs in order to explain how a decision was made or why a certain result was achieved [3].

\section{Methodology and Experiment}

We have collected a total of 46 facial palsy images and 21 normal face images. The resolutions of the facial palsy images and normal face images were about $691 \times 1024$ and $150 \times 180$, respectively. The facial palsy images were obtained from Mater Misericordiae Health Services in Brisbane. These images show facial weaknesses, such as not being able to close both eyes, twitching at the corner of mouth when smiling, and asymmetry cheeks. The normal images were obtained from Asian Emotion Database [45]. The database is arranged into train and test data sets separately. Firstly, the images are graded manually into five scales using the HouseBrackmann grades. Due to the insufficient number of images, we are not able to obtain grade VI images.

Figure 1 illustrates the overall process of the automated House-Brackmann Grading system for face images. To remove noises, a 2D Gaussian blur filter is applied on the sample face images. The images are then converted into gray scale images and normalized using a Histogram equalization process.

An automated face-area detector is developed using the Haar Cascade classifier [46] implanted on Open CV (Open Source Computer Vision: http://opencv.org) API. Haar Cascade classifier combines Ada boost algorithm for feature selection and a cascade of classifiers for rapid scanning of face images. The face-area detector automatically locates a face in a sample image and places a region of interest (ROI) rectangle. The detec- tor is calibrated such that it detects an ROI rectangle area that encloses the far edges of the eyes, the top of the eye brows, and the bottom of the lower lip. For the training data sets, the rectangle is manually rotated and/or moved to center so that it faces inside the rectangle and that the center line of the rectangle passes through the center of the face, i.e., between the eyes and the center of nose. The rectangle regions of the face images are then extracted, rescaled, and down sampled to $100 \times 100$ pixel square gray-scale images.

Figure 2 shows the intermediate images of normal face. Figure 3 shows intermediate palsy images with asymmetry mouth. For ethical reasons, these images cannot be published. Thus, sample images from the Internet of facial palsy patients are used instead. The face edges are extracted using a Sobel edge detector. Figure 2(c) and Figure 3(c) show the differences between left and right faces when we fold the faces symmetrically. The differences are measured using Hamming Distance [47], i.e., the number of positions at which the corresponding pixels are different. Therefore, each image is represented by 100 Hamming distance values.

Principal component analysis (PCA) is performed on the Hamming distance features and the top-ten principal components are selected as another set of features of the sample data sets. To compute the principal components, the Hamming distance features of face images are represented in a 100 by 67 matrix ( 100 rows and 67 columns), where each column is the Hamming distance features of a face image. Eigenvectors are computed using Open CV API. The top ten eigenvectors are selected by sorting them in descending order of their eigenvalues. The 5 groups of images are then classified by 5 -ary classifiers. .

\section{Experimental Results}

This section showcases results of the experiments. In a Receiver Operating Characteristic (ROC) curve, the sensitivity (true positive rate) is plotted as a function of specificity (false positive rate) for different cut-off points. A test with perfect discrimination (no overlap in the two distributions) has a ROC plot that passes through the upper left corner. Therefore the closer the ROC plot is to the upper left corner, the higher the overall accuracy of 
the test[48]. Figure 4(a) shows the ROC curve of SVM with ten principal components, the area under the curve is 0.73 . Figure 4 (b) shows the ROC curve of Hamming distance with SVM which gives better results of about 0.87 . It provides good curve for palsy data as shown.

Accuracy may not be a sufficient measure to evaluate the performance of classifiers. A classifier may be able to obtain a very high accuracy by classifying all test results to majority class but classifying wrongly the minority class. Therefore, the use of accuracy and mean square error rate are inappropriate for imbalanced dataset like this emotion dataset. So we adopted the use of other kinds of evaluation metrics to measure the classifiers' capability to differentiate the two-class problem under the case of imbalanced data distribution. They are namely, F-measure and Geometric Means Measure (GMM), in which the performance measures are independent to prior probabilities. F-measure is a metric derived from recall and precision. Some variants would make the weighting equal as it is considered that the occurrences of false positive and false negative are likely equal. According to the evaluations by Barandela et al.[49], GMM is a more appropriate metric to evaluate the classifier performance on the datasets which are imbalanced distribution. Both F-measure and GMM are good indicators as they maximize the accuracy on each of the two classes while keeping these accuracies balanced. The GMM is defined as the square root of the product of accuracy on positive samples and negative samples. Table 1 shows the results of using ESOM classifier on the principal component features. The results are encouraging; the F-measure is above $72 \%$. Especially in the case of Grade II, III, and IV, the results are above $90 \%$.

The 5-ary classifiers of SVM and ESOM outputs House-Brackmann scores of patients very accurately. Doctors use the scores and the characteristics of House-Brackmann grades to explain patients' facial palsy symptoms. Furthermore, the learned model parameters can be analyzed to select informative features, and apply filter to visualize regions of interest by selecting subsets of more relevant and reliable features for each case as discussed in [3].

\section{Conclusion}

In this paper, we propose to examine facial nerve palsy using SVM and Emergent SelfOrganizing Map (ESOM) and grade the degree of nerve damage using House-Brackmann score. Traditional diagnosis involves a medical doctor thoroughly examining and recording the history of the patient and determining the onset of paralysis, the rate of progression and etc. The use of an automated grading system significantly reduces the duration of the medical examination and increases consistency, because all palsy images are stored to provide a benchmark referencing and for comparative purposes. The results are encouraging. It revealed that that by using the face symmetry Hamming distance combined with Emergent Self-Organizing Map, it is able to achieve a classification rate of up to $95 \%$.

For further research, it is expected that the cellphone will be trailed as a mobile facial palsy assessment tool. This can provide timely diagnosis to patients who are located faraway from medical centers.Unlike existing medical diagnostics, such as contact thermal imaging, our proposed approach is to utilize the cell phones and infrastructure to provide a robust, noninvasive, and rapid assessment method based on the simple analysis of images. The developed system can be rapidly deployed to many rural communities over the Internet. It is reported that within the next five years, most Africans will have cell phones. Our low-cost cell phone-based data collection and diagnostic systems can be used to construct a world-wide database of diagnostic images and cases to discover the causes of diseases and patterns of disease development in developing countries.

\section{Acknowledgement $l$}

This work was supported by JCU Singapore Research Grant JCUS/003/2011/IS and a grant from the Bill \& Melinda Gates Foundation through the Grand Challenges Explorations Initiative (Grant Number: OPP1032125).

\section{References}

[1] I. Song, D. Dillon, T. J. Goh, and M. Sung, "A Health Social Network Recommender System," 
in Agents in Principle, Agents in Practice - 14th International Conference, PRIMA 2011, 2011, pp. 361-372.

[2] DSM-IV-TR, Diagnostic and Statistical Manual of Mental Disorders, Text Revision, 4th ed.: American Psychiatric Association, 2000.

[3] I. Song and N. V. Marsh, "Anonymous Indexing of Health Conditions for a Similarity Measure," Information Technology in Biomedicine, IEEE Transactions on, vol. 16, pp. 737-744, 2012.

[4] C. A. Kasse, R. G. Ferri, E. Y. C. Vietler, F. D. Leonhardt, J. R. G. Testa, and O. L. M. Cruz, "Clinical data and prognosis in 1521 cases of Bell's palsy,' International Congress Series, vol. 1240, pp. 641-647, 2003.

[5] MedicineNet.Inc.(1996).MedicineNet.com. Available: http://www.medterms.com/script/main/ art.asp? articlekey $=6556$

[6] S. Ghali, A. MacQuillan, and A. O. Grobbelaar, "Reanimation of the middle and lower face in facial paralysis: Review of the literature and personal approach," Journal of Plastic, Reconstructive \& Aesthetic Surgery, vol. In Press, Corrected Proof, 2010.

[7] A.D.A.M. Medical Encyclopedia.(2010). Bell's palsy. Available: http://www.ncbi.nlm.nih.gov/pubmedhealth/ PMH0001777/

[8] J. P. Browder, "Facial paralysis in children," Journal of Ear Nose Throat vol. 57, pp. 278-83, 1978.

[9] J. W. House and D. E. Brackmann, ”Facial nerve grading system," Otolaryngol Head Neck Surg, vol. 93 pp. 146-147, 1985.

[10] J. T. Vrabec, D. D. Backous, H. R. Djalilian, P. W. Gidley, J. P. Leonetti, S. J. Marzo, et al., "Facial Nerve Grading System 2.0," Otolaryngology - Head and Neck Surgery, vol. 140, pp. 445-450, 2009.

[11] C. Chang and C. Lin, $\{$ LIBSVM $\}$ : a library for support vector machines, 2001.

[12] A. Ultsch and M. F., "ESOM-Maps: tools for clustering, visualization, and classification with Emergent SOM," University of Marburg Dept. of Mathematics and Computer Science, Germany 2005.

[13] B. Lei, I. Song, and S. A. Rahman, "Optimal watermarking scheme for breath sound," in Neural Networks (IJCNN), The 2012 International Joint Conference on, 2012, pp. 1-6.
[14] J. Vong, J. Fang, and I. Song, "Delivering financial services through mobile phone technology: a pilot study on impact of mobile money service on micro-entrepreneurs in rural Cambodia," International Journal of Information Systems and Change Management, vol. 6, pp. 177-186, 2012.

[15] D. M. Angaran, 'Telemedicine and telepharmacy: Current status and future implications," American Journal of Health-System Pharmacy, vol. 56, pp. 1405-1426, Jul 1999.

[16] D. M. Hilty, J. S. Luo, C. Morache, D. A. Marcelo, and T. S. Nesbitt, "Telepsychiatry - An overview for psychiatrists," Cns Drugs, vol. 16, pp. 527-548, 2002.

[17] D. M. Hilty, S. L. Marks, D. Urness, P. M. Yellowlees, and T. S. Nesbitt, "Clinical and educational telepsychiatry applications: A review," Canadian Journal of Psychiatry-Revue Canadienne De Psychiatrie, vol. 49, pp. 12-23, Jan 2004.

[18] P. Yellowlees, M. M. Burke, S. L. Marks, D. M. Hilty, and J. H. Shore, 'Emergency telepsychiatry," Journal of Telemedicine and Telecare, vol. 14 , pp. 277-281, 2008

[19] R. Harrison, W. Clayton, and P. Wallace, "Can telemedicine be used to improve communication between primary and secondary care?," British Medical Journal, vol. 313, pp. 1377-1381, 1996

[20] C. A. Frantzidis, C. Bratsas, M. A. Klados, E. Konstantinidis, C. D. Lithari, A. B. Vivas, et al., "On the Classification of Emotional Biosignals Evoked While Viewing Affective Pictures: An Integrated Data-Mining-Based Approach for Healthcare Applications," Information Technology in Biomedicine, IEEE Transactions on, vol. 14 , pp. 309-318, 2010

[21] L. Tang, X. Zhou, Z. Yu, Y. Liang, D. Zhang, and H. Ni, "MHS: A multimedia system for improving medication adherence in elderly care," IEEE Systems Journal, vol. 5, pp. 506-517, 2011

[22] B. Elvevag, P. W. Foltz, M. Rosenstein, and L. E. DeLisi, "An automated method to analyze language use in patients with schizophrenia and their first-degree relatives," Journal of Neurolinguistics, vol. 23, pp. 270-284, 2009.

[23] AD Tilaka, J Diederich, I Song, and A. Teoh, "Automated Method for Diagnosing Speech and Language Dysfunction in Schizophrenia," in Mental Health Informatics, I. S. Margaret Lech, Peter Yellowlees, and Joachim Diederich, Ed., ed: Springer, 2013.

[24] L. S. A. Low, N. C. Maddage, M. Lech, L. B. Sheeber, and N. B. Allen, "Detection of clinical 
depression in adolescents' speech during family interactions," IEEE Transactions on Biomedical Engineering, vol. 58, pp. 574-586, 2011.

[25] B. Lei, I. Song, and S. A. Rahman, "Robust and secure watermarking scheme for breath sound," Journal of Systems and Software, 2013.

[26] R. Cowie, E. Douglas-Cowie, N. Tsapatsoulis, G. Votsis, S. Kollias, W. Fellenz, et al., "Emotion recognition in human-computer interaction," IEEE Signal Processing Magazine, vol. 18, pp. 32-80, 2001.

[27] Y. Yacoob and L. S. Devis, "Recognizing human facial expressions from long image sequences using optical flow," IEEE Transactions on Pattern Analysis and Machine Intelligence, vol. 18, pp. 636-642, 1996.

[28] T. Otsuka and J. Ohya, "Recognition of facial expressions using HMM with continuous output probabilities," 1996, pp. 323-328.

[29] M. Pantic and L. . M. Rothkrantz, "Automatic analysis of facial expressions: The state of the art," IEEE Transactions on Pattern Analysis and Machine Intelligence, vol. 22, pp. 1424-1445, 2000.

[30] P. Ekman, E. L. Rosenberg, and M. Heller, "What the face reveals. Basic and applied studies of spontaneous expression using the facial action coding system (FACS)," Psychotherapies, vol. 18, pp. 179-180, 1998.

[31] M. F. Valstar and M. Pantic, "Fully Automatic Recognition of the Temporal Phases of Facial Actions," IEEE Transactions on Systems, Man, and Cybernetics, Part B: Cybernetics, 2011.

[32] R. T. Stone and C. S. Wei, "Exploring the linkage between facial expression and mental workload for arithmetic tasks," 2011, pp. 616-619.

[33] M. Nambu, K. Nakajima, M. Noshiro, and T. Tamura, "An algorithm for the automatic detection of health conditions," Engineering in Medicine and Biology Magazine, IEEE, vol. 24, pp. 38-42, 2005.

[34] V. Vapnik and A. Lerner, "Pattern Recognition using Generalized Portrait Method," Automation and Remote Control, vol. 24, 1963.

[35] B. E. Boser, I. M. Guyon, and V. N. Vapnik, "A training algorithm for optimal margin classifiers," in Proc. Fifth Annual Workshop on Computational Learning Theory, 1992, pp. 144-152.
[36] C. Cortes and V. Vapnik, "Support-vector networks," Machine Learning, vol. 20, pp. 273-297, 1995.

[37] C. J. C. Burges, ”A tutorial on support vector machines for pattern recognition," Data mining and knowledge discovery, vol. 2, pp. 121-167, 1998.

[38] T. Joachims, "Text categorization with support vector machines: Learning with many relevant features," Machine learning: ECML-98, pp. 137$142,1998$.

[39] J. D. M. Rennie, 'Improving multi-class text classification with naive Bayes," Massachusetts Institute of Technology, 2001.

[40] T. Kohonen, "The self-organizing map," Proceedings of the IEEE, vol. 78, pp. 1464-1480, 1990.

[41] A. Ultsch, "Self-organizing neural networks for visualization and classification," 1993.

[42] A. Ultsch, "Maps for the visualization of highdimensional data spaces," in Proc. Workshop on Self organizing Maps, 2003, pp. 225-230.

[43] A. Ultsch, U*-matrix: a tool to visualize clusters in high dimensional data: Fachbereich Mathematik und Informatik, 2003.

[44] A. Ultsch, "Clustering with SOM: U*C," presented at the WSOM, 2005.

[45] J.-J. Wong and S.-Y. Cho, "A Support Vector Reduced Multivariate Polynomial Model for Face Emotion Recognition," IEEE Transactions on Information Forensics and Security, 2007.

[46] P. Viola and M. Jones, "Rapid object detection using a boosted cascade of simple features," in Computer Vision and Pattern Recognition, 2001. CVPR 2001. Proceedings of the 2001 IEEE Computer Society Conference on, 2001, pp. I-511-I518 vol. 1.

[47] Hamming and R. W., "Error detecting and error correcting codes," Bell System Technical Journal, vol. 29, pp. 147-160, 1950 .

[48] M. Maloof, "Learning when data sets are imbalanced and when costs are unequal and unknown," in Proceedings of the ICML, Workshop on Learning from Imbalanced Data Sets, 2003.

[49] R. Barandela, J. S. Snchez, V. Garca, and E. Rangel, "Strategies for learning in class imbalance problems," Pattern Recognition, vol. 36, pp. 849-851, 2003. 